This is the author's copy of the publication as archived with the DLR's electronic library at http://elib.dlr.de. Please consult the original publication for citation.

\title{
Energy-Based Cooperative Control for Landing Fixed-Wing UAVs on Mobile Platforms Under Communication Delays
}

T. Muskardin; A. Coelho; E. R. D. Noce; A. Ollero; K. Kondak

\section{Copyright Notice}

(C)2020 IEEE. Personal use of this material is permitted. However, permission to reprint/republish this material for advertising or promotional purposes or for creating new collective works for resale or redistribution to servers or lists, or to reuse any copyrighted component of this work in other works must be obtained from the IEEE.

\section{Citation Notice}

@ARTICLE\{muskardin2020energy,

author $=\{$ T. \{Muskardin\} and A. \{Coelho\} and E. R. D. \{Noce\} and A. \{01lero\} and K. \{Kondak\}\},

journal $=\{$ IEEE Robotics and Automation Letters $\}$,

title $=\{$ Energy-Based Cooperative Control for Landing Fixed-Wing UAVs on Mobile Platforms Under Communication Delays $\}$,

year $=\{2020\}$,
volume $=\{5\}$

volume $=\{5\}$

number $=\{4\},-5088\}$

doi $=\{10.1109 /$ LRA. $2020 \cdot 3005374\}\}$

[1] T. Muskardin, A. Coelho, E. R. D. Noce, A. Ollero, and K. Kondak. Energy-based cooperative control for landing fixed-wing uavs on mobile platforms under communication delays. IEEE Robotics and Automation Letters, 5(4):5081-5088, 2020. doi:10.1109/LRA.2020.3005374. 


\title{
Energy-Based Cooperative Control for Landing Fixed-Wing UAVs on Mobile Platforms under Communication Delays
}

\author{
Tin Muskardin ${ }^{1}$, Andre Coelho $^{1}$, Eduardo Rodrigues Della Noce ${ }^{1}$, Anibal Ollero ${ }^{2}$, \\ and Konstantin Kondak ${ }^{1}$
}

\begin{abstract}
The landing of a fixed-wing UAV on top of a mobile landing platform requires a cooperative control strategy, which is based on relative motion estimates. These estimates typically suffer from communication or processing time delays, which can render an otherwise stable control system unstable. Such effects must therefore be considered during the design process of the cooperative landing controller. In this paper the application of a model-free passivity-based stabilizing controller is proposed, which is based on the monitoring of energy flows in the system, and actively dissipating any given active energy by means of adaptive damping elements. In doing so, overall system passivity and consequently stability is enforced in a straightforward and easy to implement way. The proposed control system is validated in numerical simulations for round trip delays of up to 4 seconds.
\end{abstract}

Index Terms-Field Robots, Aerial Systems: Applications, Cooperating Robots, Telerobotics and Teleoperation

\section{INTRODUCTION}

$\mathbf{L}$ ANDING an autonomous unmanned aerial vehicle (UAV) on top of a mobile platform has been the subject of extensive research in recent years [1]-[11]. Benefits of such approach include the increase of payload capacity by removing the landing gear, the simplification of landing procedures in the presence of strong cross winds or in the absence of proper runways, or the use as fail-safe system in case of landing gear malfunction [8]. The first two points are especially relevant for High Altitude Platforms (HAP) or High Altitude Long Endurance (HALE) aircraft, given their high weight criticality and slow landing airspeeds. Among the proposed approaches, the German research project Airport2030 [1] and the European project GABRIEL [2] aimed at providing solutions for the commercial aviation sector. Such systems could provide significantly higher levels of safety and efficiency and have already caught the attention of the aviation industry. On the other hand, the idea of landing Micro Air Vehicles (MAVs) on

Manuscript received: February, 24, 2020; Revised May, 20, 2020; Accepted June, 10, 2020.

This paper was recommended for publication by Editor Jonathan Roberts upon evaluation of the Associate Editor and Reviewers' comments. This work was partially supported by the European Commission FP7 ICT Programme under the project EC-SAFEMOBIL 288082.

${ }^{1}$ Tin Muskardin, Andre Coelho, Eduardo Rodrigues Della Noce, and Konstantin Kondak are with the German Aerospace Center DLR, Institute of Robotics and Mechatronics, 82234 Wessling, Germany tin.muskardinedlr.de

${ }^{2}$ Anibal Ollero is with the University of Seville, ETS Ingenieria, Camino Descubrimientos s/n, 41092 Sevilla, Spain aollero@us.es

Digital Object Identifier (DOI): see top of this page.

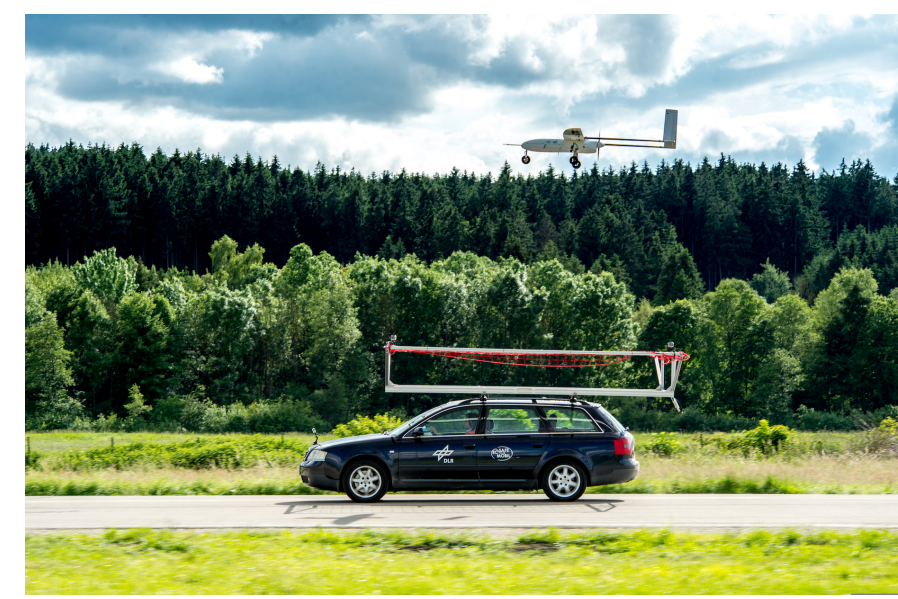

Fig. 1: Demonstrator setup for landing experiments.

moving platforms has also been documented in several works, such as [4], [7], [10], [12]. To cite a few, in [4] fiducial markers are used to detect the landing platform, enhancing the measurements with a sensor fusion approach based on an unscented Kalman filter. In [6] a PID controller is utilized while applying commercially available, low-cost sensors and algorithms combined in a Kalman filter for relative position and velocity estimation. In [9] the same task is performed without any additional infrastructure, relying on multirotor on-board sensing (IMU, altitude sensors, and RGB camera) and applying Deep Deterministic Policy Gradients (DDPG). In [10] an extended visual-inertial odometry framework for SLAM and a suite of estimation algorithms were adapted while closing the position loop via a model-predictive controller.

Although many of the aforementioned approaches have demonstrated successful landings, additional issues remain to be solved to allow for a wider application of the cooperative landing concept. While vision-based tracking still needs to be proven robust against occlusions and variable lighting conditions, control systems relying on wireless communication channels to establish the exchange of data between the involved vehicles can have their stability characteristics jeopardized by the presence of time delays, jitter, package loss, and communication blackouts.

An alternative to cope with undesired communications phenomena consists in decoupling the action of the two vehicles in such way, that the landing task simplifies to the problem of tracking a possibly delayed reference for each of the vehicles. 
In [8], the lateral control is performed by the UAV, while the ground vehicle (UGV) remains in the center of the runway. On the other hand, longitudinal control is performed by the UGV, while the UAV flies at constant longitudinal velocity. However, as mentioned in [13], better performance can be achieved if both vehicles are fully cooperative in order to achieve the rendezvous task. In this regard, as mentioned in [11], to achieve optimal performance and robustness, the effects of time delay should be taken into account in the controller-design process.

Although the effects of time delay and package loss have not been deeply investigated in the field of cooperative landing systems, this issue has been extensively studied in telemanipulation [14], where energy-based approaches like the Time Domain Passivity Approach (TDPA) [15] have been employed to ensure the stability of the task. Even though telemanipulation approaches cannot be directly applied to the landing scenario, a suitable adaptation would allow for the landing system to remain stable regardless of communications effects.

In light of that, this paper presents an energy-based control framework allowing for a safe operation of the fully cooperative landing system in the presence of communication delays. The effectiveness of the proposed framework with respect to ensuring system stability is validated through numerical simulation of the setup presented in Fig. 1 in the presence of round-trip delays of up to 4 seconds.

\section{Vehicle Controllers}

The cooperative landing maneuver imposes certain requirements on the aircraft control system. As mentioned in [8], classical control approaches using three separate loops for altitude, airspeed, and course control would not be capable of fulfilling the requirement of decoupled airspeed and altitude control, which can only be achieved through coordinated control inputs of elevator and throttle. A flight control strategy based on energy principles known as Total Energy Control System (TECS) is capable of providing such functionality [16].

Adding to that, TDPA-based approaches rely on monitoring and regulating the energy traffic between the two agents. It is assumed that the controlled vehicles are passive and the only possible source of energy is introduced by the position synchronization controllers in the presence of communication delays and package loss. Since the TECS controller makes the energy insertion into the flight control system evident, it is a suitable candidate to work together with the passivity controller, as will be explained in the following sections.

\section{A. Total Energy Control System}

Conventional flight control systems consist of several singleinput, single-output (SISO) control loops, where the engine is typically used for speed control and the elevator for altitude control. However, this approach does not account for the strong inherent coupling of altitude and speed responses to elevator and thrust commands, as illustrated in Fig. 2.

From the scheme in Fig. 2, it can be seen that the thrust command (via throttle) can be used for adjusting the total

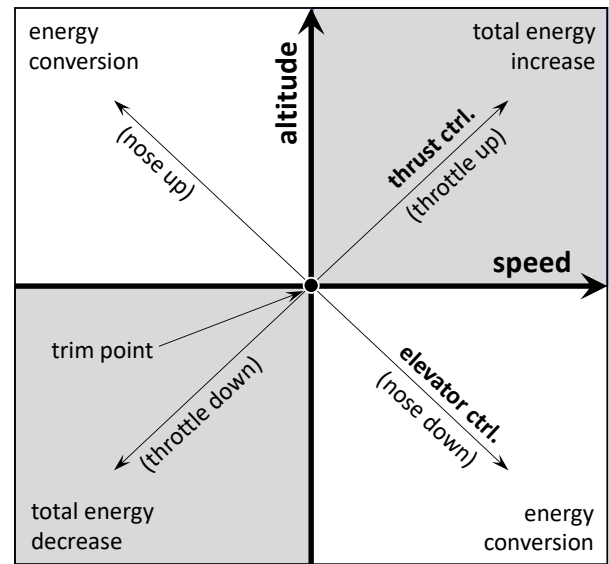

Fig. 2: Short-term aircraft control responses. Throttle controls the total energy insertion while elevator controls the potentialkinetic energy distribution.

energy of the aircraft while the pitch command (via elevator) regulates the energy exchange. Therefore, since no bilateral altitude synchronization between the vehicles is assumed (only aircraft controls altitude), the energy introduced into the system by the (horizontal) position synchronization controller due to communication delays and package loss can be monitored and regulated through the port $\left\langle V_{k, a}^{\text {des }}, T_{a}^{c m d}\right\rangle$, where $V_{k, a}^{\text {des }}$ is the desired aircraft (index a) ground speed and $T_{a}^{c m d}$ is the thrust commanded by the TECS in order to change the total energy of the system.

For the lateral-directional control of the aircraft, a conventional cascaded SISO controller using the ailerons as actuators was implemented. Rudder control was not used, since the natural directional stability of the aircraft is sufficiently fast to drive the sideslip angle back to zero after a disturbance. In its energetic behavior, the lateral directional controller plays a similar role as the pitch controller, i.e. it does not introduce energy into the system, but rather acts within an energy conservation layer. Therefore, no additional lateral passivity controller needs to be added in the presence of delay.

\section{B. Ground Vehicle Control}

For ground vehicle control, a simple autopilot system was developed. Two independent SISO controllers were used for each direction of motion. For the longitudinal direction, a simple PI-controller was sufficient to accurately control ground speed, while for the lateral direction a P-controller was sufficient for accurate course control [8]. Similar to the analysis for the UAV, it can be noted that the lateral controller does not introduce energy to the system by changing the course angle of the vehicle only. The energy insertion port of the ground vehicle consists of the flow and effort pair $\left\langle V_{k, g}^{\text {des }}, T_{g}^{c m d}\right\rangle$, which corresponds to the desired ground vehicle (index g) velocity $V_{k, g}^{d e s}$ and commanded engine thrust $T_{g}^{c m d}$.

\section{BILATERAL POSITION SYNCHRONIZATION}

As previously mentioned, a bilateral position synchronization control structure has been applied in order to enhance the performance of the landing controller. Bilateral here implies 


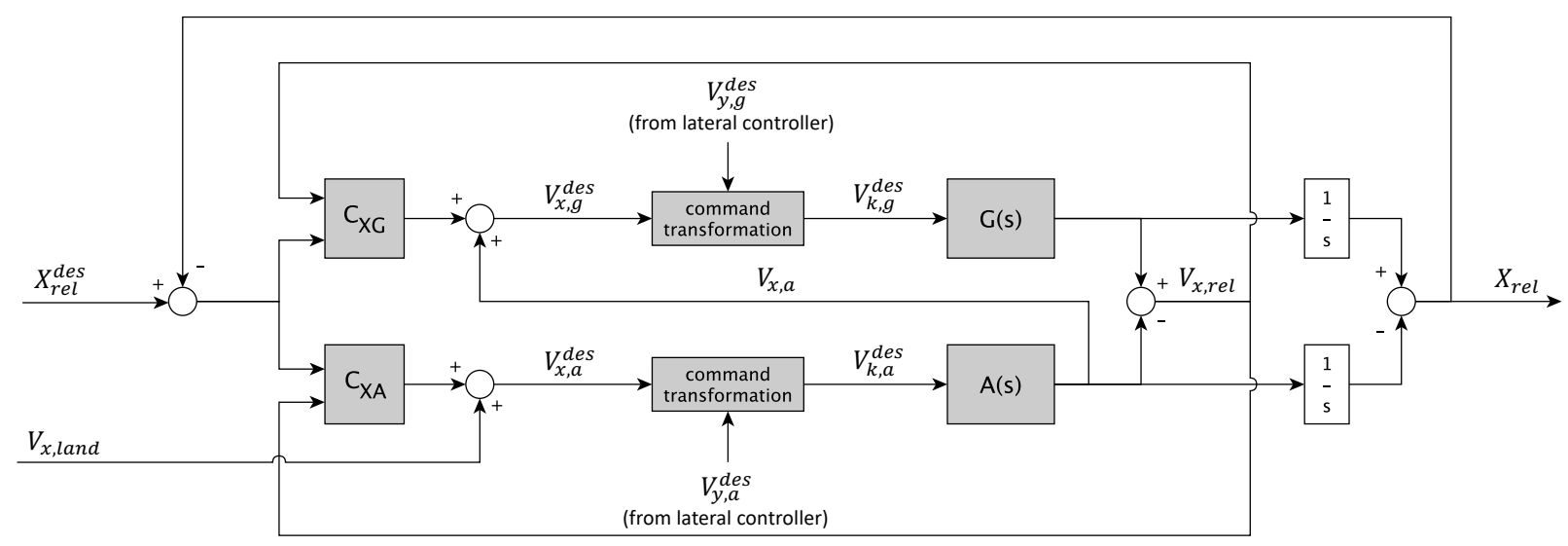

Fig. 3: Bilateral longitudinal position synchronization controller with velocity controlled ground vehicle $\mathrm{G}(\mathrm{s})$ and aircraft $\mathrm{A}(\mathrm{s})$. The subscripts $a$ and $g$ refer to aircraft and ground vehicle related quantities, respectively.

that per motion direction, both vehicles actively contribute to achieve a rendezvous. For the case where each vehicle takes care of one direction as shown in [8], the strategy is considered unilateral, even though the vehicles would still cooperate to achieve the landing.

Fig. 3 shows the block diagram for the longitudinal position synchronization controller in absence of communication delays. The longitudinal cooperative position synchronization controllers $C_{X G}$ and $C_{X A}$ are PID controllers on the relative desired position $X_{r e l}^{\text {des }}$ along the runway, whose outputs are calculated in terms of inertial runway axes (i.e. Cartesian coordinates) instead of ground speed vector magnitudes along the vehicle's kinematic $\mathrm{x}$-axes, as used in [8]. A subsequent transformation into the spherical command structure $\left(V_{k}\right.$ and $\chi)$ as expected by the lower-level vehicle controllers is performed in the blocks command transformation as shown in (1). Also, only the x-component of the aircraft velocity is fed back to the UGV instead of the velocity vector magnitude $V_{k}$.

$$
V_{k}=\sqrt{V_{x}^{2}+V_{y}^{2}}, \quad \chi=\arctan \left(\frac{V_{y}}{V_{x}}\right) .
$$

As can also be seen in Fig. 3, the aircraft controller receives the desired landing speed command $V_{x, \text { land }}$ as feedforward term, while the ground vehicle controller receives the current UAV $\mathrm{x}$-axis velocity as feedforward term. Apart from that, both control architectures are symmetric. The lateral controller also has a similar architecture, with the desired lateral landing speed usually being set to zero. Apart from that, the controlled vehicles $A(s)$ and $G(s)$ receive course commands instead of velocity commands [13].

Due to the fact that the only energy introduction ports, $\left\langle V_{k, a}^{\text {des }}, T_{a}^{\text {cmd }}\right\rangle$ and $\left\langle V_{k, g}^{\text {des }}, T_{g}^{\text {cmd }}\right\rangle$ belong to the vehicles' longitudinal controllers, the remainder of this paper will mainly focus on those ports and their behavior in the presence of delay and package loss. The reader is referred to [13] for an in-depth discussion of the entire application.

\section{Time-Delay-Robust Landing Control}

It is well known that the presence of time delay and package loss in the feedback loops of the landing control system is a possible cause of instability. As shown in [8], the physical delays due to wireless communication typically range in the area of $50 \mathrm{~ms}$ and less, but the overall signal routing and processing can induce higher delays. In extreme cases, where large amounts of data (e.g. live telemetry and video streaming) are being transferred over the same communication channel, or where range limits are being reached and high package loss is present, even temporary high delays of more than $5 \mathrm{~s}$ are possible. In order to handle those situations, the presence of a fail-safe mechanism to guarantee system stability is required. The control strategy chosen in this work is an adaptation of the Time Domain Passivity Control Approach, which is based on monitoring energy flows in the system and actively dissipating any given active energy by means of adaptive damping elements [15], [17]. Throughout this section the concept will be presented and a description of its application to the landing control system will be provided. A performance analysis demonstrating its behavior in the presence of high time delays will be presented in Section V.

\section{A. Time Domain Passivity Control Approach}

TDPA was first developed in the field of teleoperation, where a remote manipulator (e.g. in space [14]) is operated via a delayed communication channel by a human operator, who receives haptic feedback based on the remote task. For cases where position synchronization is desired, as for the coordination of two agents, a Position-Position (P-P) architecture [18] can be applied. Even though a traditional P-P architecture cannot be directly applied to the landing scenario, an overview will be given in order to facilitate the understanding of the proposed architecture.

Position-Position Architecture: The block diagram of a Position-Position architecture is illustrated in Fig. 4. The signals $f_{m}$ and $f_{s}$ represent the computed master and slave forces, $v_{m}$ and $v_{s}$ the master and slave velocities, $f_{h}$ and $f_{e}$ the forces applied by the human operator and the environment (for the traditional teleoperation case), and $T_{f}$ and $T_{b}$ the forward and backward time delays. The delayed signals $v_{s d}$ and $v_{m d}$ 


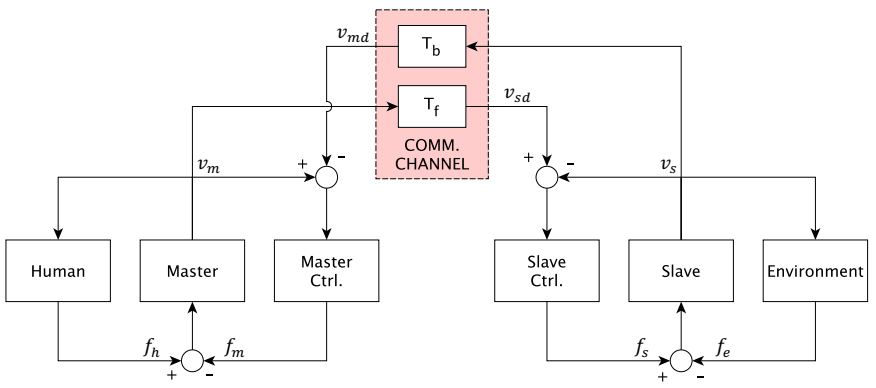

Fig. 4: Block diagram of P-P teleoperation architecture.

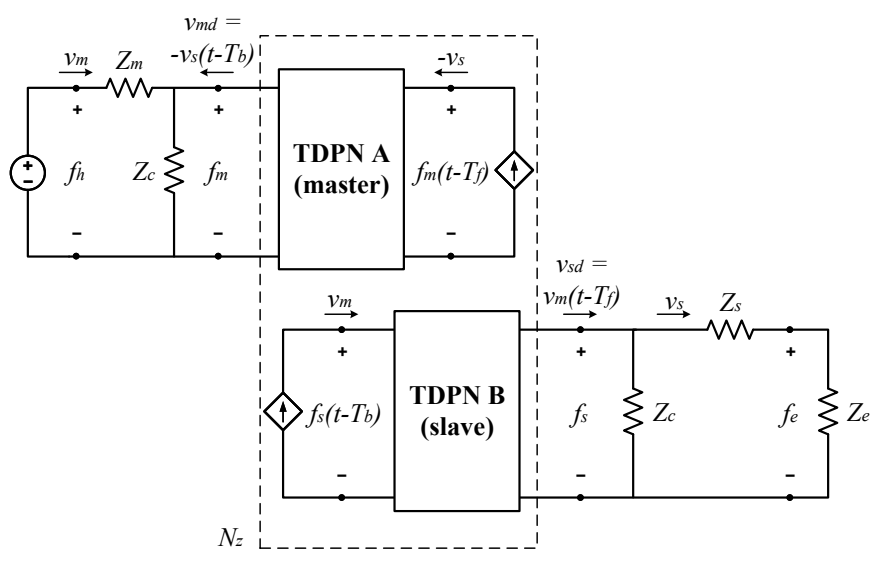

Fig. 5: Circuit representation of P-P teleoperation architecture with TDPN-augmented communication network.

are defined as follows

$$
v_{s d}(t)=v_{m}\left(t-T_{f}\right), \quad v_{m d}(t)=v_{s}\left(t-T_{b}\right)
$$

where $v_{m d}$ and $v_{s d}$ represent the desired master and slave velocities respectively. The master and slave controllers are typically implemented as PD-controllers on position.

As can be seen in Fig. 4, the block diagram of the P-P architecture does not provide sufficient information about the causality of the communication channel in terms of energy flows, which is necessary for the application of TDPA. Therefore, the circuit representation of the P-P architecture, where the communication channel is represented by a pair of TimeDelay Power Networks (TDPNs, [15]), is introduced as shown in Fig. 5. The port variables for the master side TDPN are now found as $f_{m}(t)$ and $v_{s}\left(t-T_{b}\right)$ at the left port, while the variables at the right port include $f_{m}\left(t-T_{f}\right)$ and $v_{s}(t)$. The port variables for the slave side TDPN are $f_{s}\left(t-T_{b}\right)$ and $v_{m}$ at the left port, while the variables at the right port include $f_{s}(t)$ and $v_{m}\left(t-T_{f}\right)$. It should also be noted that the exchanged force signals have no direct impact on the vehicle's motion control systems, but are used for passivity control purposes only.

Once the correct network representation of the system is found, the application of TDPA for the passivation of the communication network is straightforward..

Time Delay Power Networks: The introduction of TDPNs in Fig. 5 allows for investigating the stability of the teleoperation system by analyzing the energy flows at the ports of the TDPN. Fig. 6 illustrates the energy flow through the TDPN, where $E^{M}$ and $E^{S}$ are the energies evaluated on the left (master) and right (slave) hand side of the TDPN respectively, while $f_{1}(k), v_{1}(k)$,

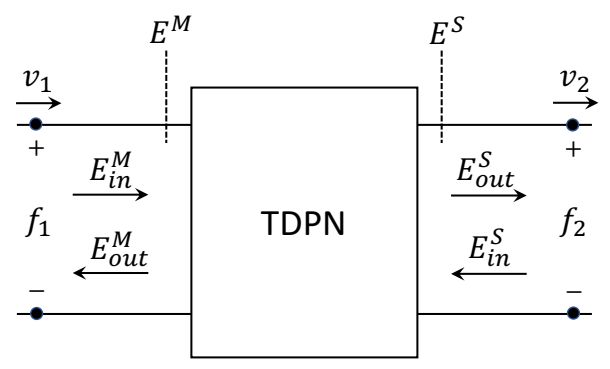

Fig. 6: Energy flow through the TDPN.

and $f_{2}(k), v_{2}(k)$ refer to the force and velocity pairs at instant $k$ on the left and right ports.

As shown in [15], the TDPN will be passive if:

$$
E^{N}(k)=E^{M}(k)+E^{S}(k) \geq 0, \quad \forall k \geq 0,
$$

where

$$
\begin{gathered}
E^{M}(k)=E_{\text {in }}^{M}(k)-E_{\text {out }}^{M}(k)=\Delta T \sum_{j=0}^{k} f_{1}(j) v_{1}(j), \\
E^{S}(k)=E_{\text {in }}^{S}(k)-E_{\text {out }}^{S}(k)=\Delta T \sum_{j=0}^{k}-f_{2}(j) v_{2}(j) .
\end{gathered}
$$

$E_{\text {in }}^{M}(k), E_{\text {out }}^{M}(k), E_{\text {in }}^{S}(k)$, and $E_{\text {out }}^{S}(k)$ represent the energy flows into and out of the TDPN at time step $k$ on the master and slave side respectively. These quantities constitute monotonically increasing and positive-definite functions, which can be calculated as follows

$$
E_{i n}^{M}(k)= \begin{cases}\Delta T f_{1}(k) v_{1}(k)+E_{i n}^{M}(k-1), & \text { if } f_{1}(k) v_{1}(k)>0 \\ E_{\text {in }}^{M}(k-1), & \text { else }\end{cases}
$$

$E_{\text {out }}^{M}(k)= \begin{cases}-\Delta T f_{1}(k) v_{1}(k)+E_{\text {out }}^{M}(k-1), & \text { if } f_{1}(k) v_{1}(k)<0 \\ E_{\text {out }}^{M}(k-1), & \text { else. }\end{cases}$

$E_{\text {in }}^{S}$ and $E_{\text {out }}^{S}$ are computed in a similar manner using $f_{2}$ and $v_{2}$. Unfortunately, (3) cannot be directly evaluated in a real (delayed) system, since the real-time values of the energies at either side of the TDPN are not available at the same time. It is therefore necessary to observe the energy flows at each port individually, which can be accomplished by taking the direction of the energy flows into account. Therefore, (3) can be rearranged as follows.

$$
\begin{aligned}
& E_{\text {obs }}^{L 2 R}(k)=E_{\text {in }}^{M}\left(k-T_{f}(k)\right)-E_{\text {out }}^{S}(k) \geq 0, \quad \forall k \geq 0, \\
& E_{\text {obs }}^{R 2 L}(k)=E_{\text {in }}^{S}\left(k-T_{b}(k)\right)-E_{\text {out }}^{M}(k) \geq 0, \quad \forall k \geq 0,
\end{aligned}
$$

where $E_{o b s}^{L 2 R}(k)$ represents the energy flow from left to right, observed on the right side of the TDPN, and $E_{o b s}^{R 2 L}(k)$ the energy flow from right to left, observed on the left side of the TDPN. $T_{f}(k)$ and $T_{b}(k)$ are the forward and backward delays, respectively. Since, the energy flows $E_{\text {in }}^{M}$ and $E_{\text {in }}^{S}$ are monotonic and positive defined functions, the observed energy flows from (8) and (9) are upper bounded by their actual values, guaranteeing the passivity of the delayed system. Using 
(8) and (9) these relations can be more intuitively expressed as

$$
\begin{aligned}
& E_{\text {out }}^{S}(k) \leq E_{\text {in }}^{M}\left(k-T_{f}(k)\right) \quad(\text { on slave side }) \\
& E_{\text {out }}^{M}(k) \leq E_{\text {in }}^{S}\left(k-T_{b}(k)\right) \quad(\text { on master side })
\end{aligned}
$$

which implies that the flows out of the TDPN must be upper bounded by their respective input flows.

Passivity Observer and Passivity Controller: Once the appropriate observable energy flows for checking system passivity have been identified, the passivity observer (PO) and passivity controller (PC) can be defined and added to the teleoperation system. The PO will check the passivity conditions defined in (8) and (9) on each side (one PO per side) of the communication network, while taking into account previous energy dissipated by the master and slave PCs. The observed energies $W_{M}$ on the master, and $W_{S}$ on the slave side are

$$
\begin{aligned}
W_{M}(k) & =E_{\text {in }}^{S, A}\left(k-T_{b}(k)\right)-E_{\text {out }}^{M, A}(k)+E_{P C}^{M}(k-1) \\
W_{S}(k) & =E_{\text {in }}^{M, B}\left(k-T_{f}(k)\right)-E_{\text {out }}^{S, B}(k)+E_{P C}^{S}(k-1)
\end{aligned}
$$

where $E_{P C}^{M}(k-1)$ and $E_{P C}^{S}(k-1)$ are the energies dissipated by the master and slave PCs up to the previous time step. $E_{\text {out }}^{M, A}(k)$ and $E_{i n}^{S, A}\left(k-T_{b}(k)\right)$ are computed based on the pair $\left\langle v_{m d}, f_{m}\right\rangle$ and its delayed version on the master side (TDPN A), while $E_{\text {in }}^{M, B}\left(k-T_{f}(k)\right)$ and $E_{\text {out }}^{S, B}(k)$ come from the pair $\left\langle v_{s d}, f_{s}\right\rangle$ and its delayed version on the slave side (TDPN B).

The passivity controllers will be located on the vehicle side of the TDPNs and adaptively dissipate any active energy observed by the passivity observers. For the P-P architecture, two admittance-type PCs are applied in order to modify the velocity commands on both sides as

$$
\begin{gathered}
v_{m d}(k)=\hat{v}_{m d}(k)+\beta_{M}(k) f_{m}(k), \\
v_{s d}(k)=\hat{v}_{s d}(k)+\beta_{S}(k) f_{s}(k),
\end{gathered}
$$

where $\beta_{M}$ and $\beta_{S}$ represent adaptive damping coefficients for the master and slave. $\beta_{M}$ is defined as

$$
\beta_{M}(k)= \begin{cases}0 & \text { if } W_{M}(k)>0 \\ -\frac{W_{M}(k)}{\Delta T f_{m}^{2}(k)} & \text { else, if }\left|f_{m}(k)\right|>0 .\end{cases}
$$

$\beta_{S}$ is computed in an analogous manner using $W_{S}$ and $f_{s}$. The energy dissipated by the PCs will be

$$
E_{P C}^{M}(k)=\Delta T \sum_{j=0}^{k} \beta_{M}(j) f_{m}^{2}(j),
$$

and similarly for $E_{P C}^{S}(k)$. Since the cooperative control architecture used for landing does not directly match a P-P architecture, extra steps have to be taken before the TDPA can be applied.

\section{B. TDPA for Landing Control}

The first step for the application of TDPA to the cooperative control architecture presented in Section III consists in finding a suitable circuit representation to identify the correct powerconjugated pairs for passivity checking. The developed circuit representation is shown in Figs. 7 and 8 for the aircraft (index a, UAV) and ground vehicle (index g, UGV) sides, respectively.

The representation shown in Figs. 7 and 8 resembles the P-P architecture presented in the previous section, where both systems share velocity signals and have their own controllers to track the velocity of the other system received through the channel. In contrast to the P-P teleoperation concept no human operator is present in the landing application, which renders the classical master / slave assignments irrelevant, since both vehicles act as master and slave devices simultaneously in a distributed setup. In the landing context, the forward time delay $T_{f}$ refers to the communication delay from the aircraft to the ground vehicle, while the backward delay $T_{b}$ refers to the opposite direction. As previously mentioned, each vehicle transmits its current velocity through the communication channel, which serves as reference to the other vehicle's position controller.

The current ground speeds of aircraft and ground vehicle $V_{k, a}$ and $V_{k, g}$ will be referred to as $v_{a}$ and $v_{g}$ in the following, where the index $\mathrm{k}$ is dropped in favor of a concise notation. The commanded aircraft and ground vehicle forces $f_{a}$ and $f_{g}$ (corresponding to $T_{a}^{c m d}$ and $T_{g}^{c m d}$, respectively) are controllerinternal variables, also exchanged in order to monitor the energy flow of the channel. $v_{\text {land }}$ represents the desired landing velocity with respect to the ground, which is assumed to be constant and independent of the vehicle states. Consequently, $v_{\text {land }}$ is not affected by the communication channel and can be added after the passivity controller in the circuit representation as a constant current source. The overall velocity command forwarded to the vehicles thus consists of two parts - the constant landing velocity $v_{\text {land }}$, which is not a source of instability, and the velocity commands $v_{c a}$ and $v_{c g}$ provided by the vehicle's cooperative controllers, which are functions of the relative position between the vehicles. The relative position vector is calculated based on delayed data received via the communication channel, which introduces time delay, jitter, and package loss to the system. Due to its potential to cause instability, the velocity commands from the cooperative controllers must also be covered by the PO-PC. The passivity controller is represented as variable impedance $\beta$ in Figs. 7 and 8. Moreover, UAV Ctrl and UGV Ctrl represent the vehicle controllers, whose inputs are desired ground speeds.

Since saturation blocks are used in the vehicle controllers to keep the vehicles within safe velocity ranges, variable impedances $Z_{s a t}$ were added to both circuits. The velocities flowing towards the vehicle controllers after $Z_{\text {sat }}$ are therefore saturated versions of $v_{c a}+v_{g}\left(t-T_{b}\right)-v_{\text {land }}$ for the aircraft, and $v_{c g}+v_{a}\left(t-T_{f}\right)-v_{\text {land }}$ for the ground vehicle, where the position synchronization commands $v_{c a}$ and $v_{c g}$ are defined as:

$$
\begin{gathered}
v_{c a}=-v_{g}\left(t-T_{b}\right)+v_{\text {land }}+\sqrt{\left(C_{X A}\left(\hat{x}_{\text {rel }, a}\right)\right)^{2}+\left(C_{Y A}\left(\hat{y}_{\text {rel }, a}\right)\right)^{2}} \\
v_{c g}=-v_{a}\left(t-T_{b}\right)+\sqrt{\left(v_{x a}+C_{X G}\left(\hat{x}_{\text {rel }, g}\right)\right)^{2}+\left(C_{Y G}\left(\hat{y}_{\text {rel }, g}\right)\right)^{2}}
\end{gathered}
$$

where $C_{X A}, C_{Y A}, C_{X G}$, and $C_{Y G}$ refer to the cooperative position synchronization controllers on the aircraft and ground vehicle 


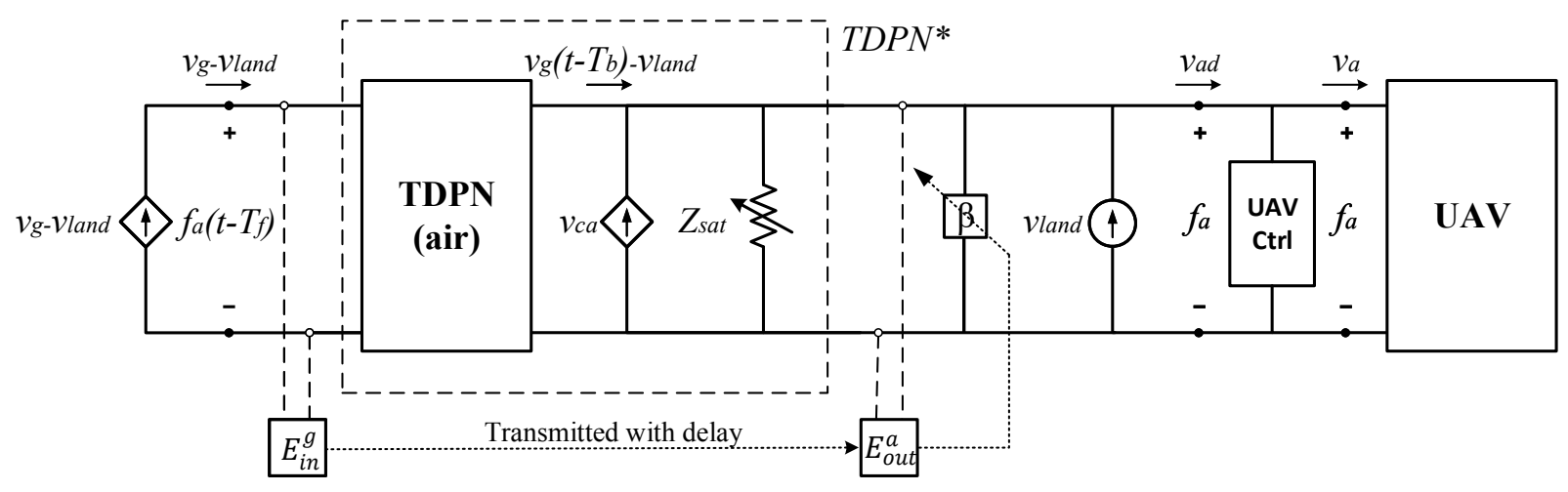

Fig. 7: Circuit representation of aircraft position synchronization controller with passivity controller and TDPN-augmented communication network representation

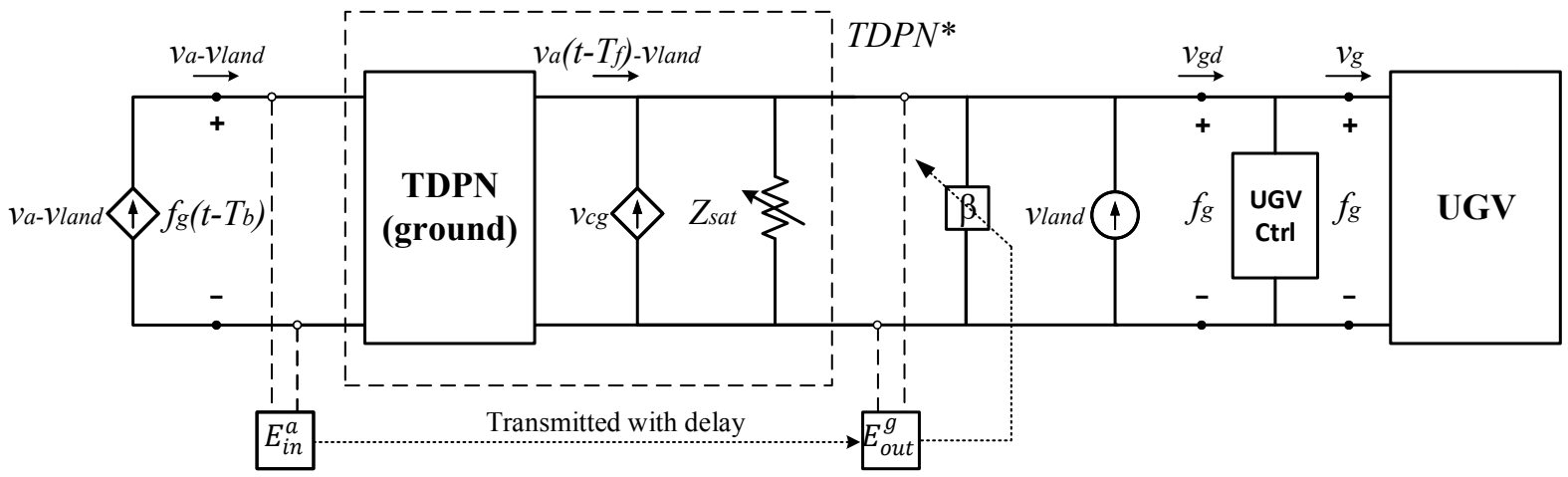

Fig. 8: Circuit representation of ground vehicle position synchronization controller with passivity controller and TDPNaugmented communication network representation

respectively, as defined in Section III.

Adding to that, $\hat{x}_{r e l, a}, \hat{x}_{r e l, g}, \hat{y}_{r e l, a}$, and $\hat{y}_{r e l, g}$ represent the estimated values of the current relative vehicle position, calculated on each vehicle individually, by extrapolating the other vehicle's delayed position [8]. The relative vehicle position estimates in the longitudinal direction are calculated as

$$
\begin{aligned}
& \hat{x}_{r e l, a}=x_{g}\left(t-T_{b}\right)+T_{b} v_{x g}\left(t-T_{b}\right)-x_{a} \\
& \hat{x}_{r e l, g}=x_{g}-x_{a}\left(t-T_{f}\right)-T_{f} v_{x a}\left(t-T_{f}\right)
\end{aligned}
$$

where analog expressions can be derived for the lateral direction. It should also be noted that full position and velocity vectors are transmitted over the channel, where all vector components are accessible after reception (e.g. $v_{x a}, v_{y a}, v_{z a}$ ), while the circuit representation shown in Figs. 7 and 8 is based on the scalar values relevant for passivity control.

As can be seen in (18) and (19), the commanded inertial velocities $v_{c a}$ and $v_{c g}$ depend on the relative vehicle states in $\mathrm{x}$ and $\mathrm{y}$ direction, thus containing both longitudinal and lateral motion information. It is therefore sufficient to monitor the scalar velocities $V_{k}$ and the applied thrust to identify excessive energy added by the communication channel. Therefore, a single PO-PC pair per vehicle is sufficient.

It is important to note that the signals $v_{g}\left(t-T_{b}\right)-v_{\text {land }}$ and $v_{a}\left(t-T_{f}\right)-v_{\text {land }}$ transmitted through the forward and backward communication channels (see Figs. 7 and 8), and relevant for passivity control, are modified on the receiving side by subtracting the first two terms of the cooperative controllers $v_{c a}$ and $v_{c g}$, as defined in (18) and (19). Only the saturated version of the last term is left to be checked by the passivity controller, before it is forwarded to the lowerlevel vehicle controllers. This way the communication channel and the position synchronization controllers are passivated. Such setup can be interpreted as an augmented TDPN (dashed boxes in Figs. 7 and 8), where the in and out energy flows are passivated by the PO-PC pairs at the TDPN ports in the same way as shown for the P-P architecture. This system architecture was chosen to allow for the adaptation of the TDPA concept to the landing control system, and does not imply any limitations regarding its applicability. It should be noted that for the undelayed case and perfectly synchronized vehicles the in and out energy pairs will match and the transmitted signals will remain untouched by the PC. One should also note that the velocities checked by the PO-PC pairs on both sides of the TDPNs represent variations of the commanded velocities around $v_{\text {land }}$. This operating point shift (subtraction of $v_{\text {land }}$ from the absolute velocity signals) allows for the distinction of inward and outward energy flows based on the sign of the power conjugated signal, as is usual in classical teleoperation setups [15]. Moreover, adding a current source to modify the desired velocity is a common practice in TDPA-based drift compensation [19], [20]. In fact, by having a part of the position synchronization controller before the passivity controller allows for automatic recovery of the drift caused by the PC. 
Force-Based Vehicle Control: As explained in the previous sections, one requirement for the application of TDPA is that power conjugated variables (velocity and force) are used as control signals. Unlike most robotic manipulators, classical aircraft and ground vehicle control systems do not allow for explicit force control, where instead velocity, course angle and flight path angle represent the available control variables. The use of the energy-based flight controller introduced in Section II, allows for retrieving the required force command. However, the ground vehicle does not allow for explicit force control, which was circumvented by using model-based force estimation. For a further improvement the use of a force observer, which would allow for more precise force estimates, could be investigated.

\section{VALIDATION}

The employed simulation setup is composed of a small UAV together with a landing platform mounted on a ground vehicle, as shown in Fig. 1. The simulation environment contains a high fidelity aircraft model (six-degrees-of-freedom rigid body with linear derivative aerodynamics), obtained by means of system identification. The same model was used to design the flight controller gains, which were validated in flight experiments and exhibited good performance without the need for re-tuning. The aircraft model can thus be considered very realistic. Atmospheric disturbances are modeled, as well as the disturbed flow field around the ground vehicle.

In order to test the effects of different communication delays on the landing system performance and the capability of the proposed approach to ensure stability in the presence of time delays, a set of landing simulations were performed with oneway communication delays varying from $0.1 \mathrm{~s}$ to $0.9 \mathrm{~s}$. In all simulations the vehicles start from the same initial condition (around $200 \mathrm{~m}$ apart) and are expected to reach zero relative position in every direction. Fig. 9 depicts the norm of the relative position vector between the vehicles for the cases where the proposed approach was inactive (top image) and active (bottom image). It can be seen that starting from a delay of $0.4 \mathrm{~s}$, the aircraft initially reaches a safety bound imposed by the landing state machine (too large deviation from landing platform center at flare initiation [13]) and issues an automatic retry before resuming the landing. Nevertheless, it can be noted that, when the passivity controller is turned off, the landing starts to take longer, up to a point (at $0.9 \mathrm{~s}$ delay) where the aircraft cannot enter the safety bounds anymore and is not able to land. On the other hand, when the proposed approach is active, it ensures that the aircraft is able to land in all simulated cases, even if an initial retry occurs for higher delays.

Moreover, in order to show how the proposed approach manipulates the commanded velocities in order to passivate the system, the desired velocities of both vehicles are depicted in Fig. 10 for a delay of $0.1 \mathrm{~s}$ and $0.9 \mathrm{~s}$. The top plots depict the desired velocities for both UAV and UGV for the nominal case $\left(T_{d}=0.1 \mathrm{~s}\right)$. It can be seen that effect of TDPA on the desired velocities (red vs. black lines) is almost negligible in this case. Therefore, the landing occurs at a very similar time as for the case when TDPA is inactive (see Fig. 9). On the
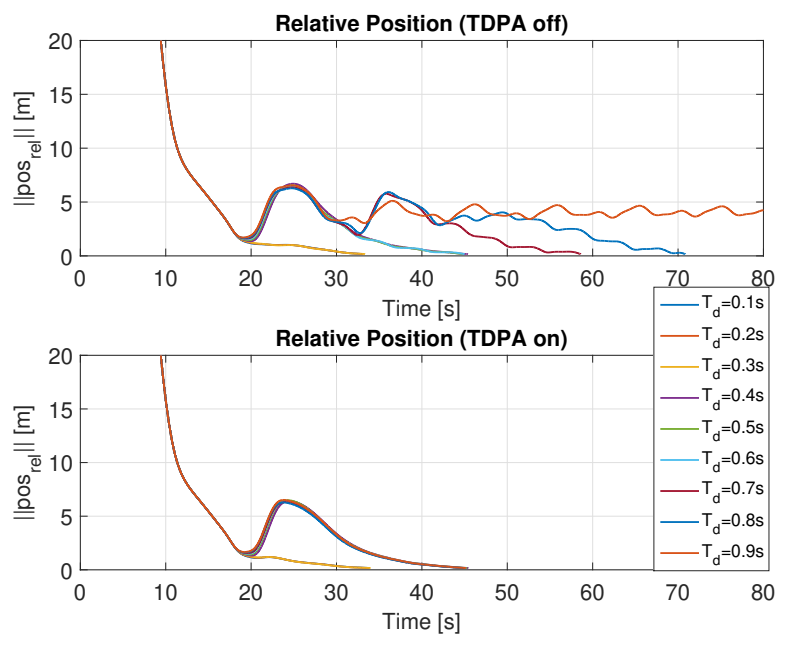

Fig. 9: Effects of time delay on the relative position of the vehicles during landing simulations. The top plot shows the norm of the relative position vector for one-way time delays varying from $0.1 \mathrm{~s}$ to $0.9 \mathrm{~s}$ without the proposed approach while bottom one shows the same quantity when the proposed approach is active.
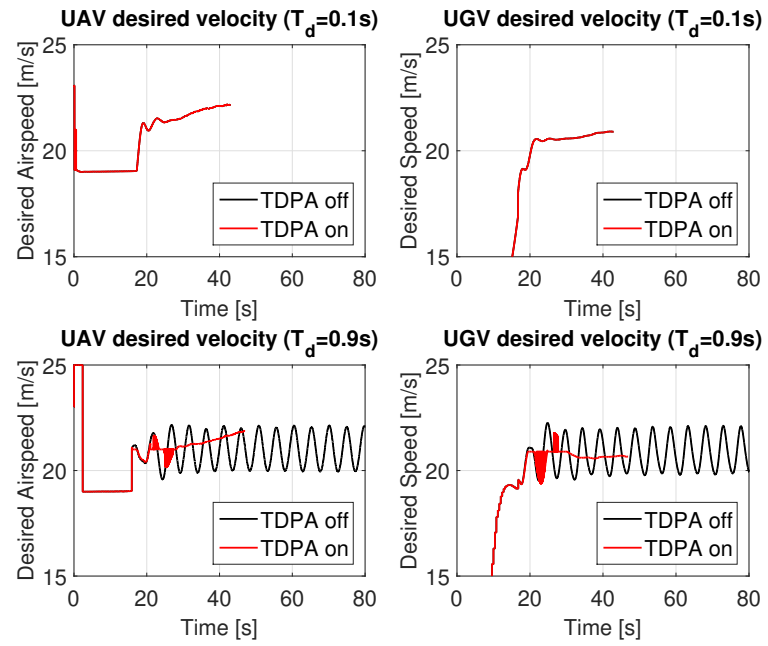

Fig. 10: Desired velocities of UAV and UGV for nominal (top) and high (bottom) time delays. For the nominal case, the effect of the proposed approach is negligible, but for high delays it modifies the velocity references and allows for a successful landing (where the red lines end). This behavior clearly illustrates the benefit of the proposed approach.

other hand, when the time delay is set to $0.9 \mathrm{~s}$ (bottom images), the commanded velocities start to oscillate when the proposed approach is not active (black lines). In that case, as seen in Fig. 9, the aircraft is unable to land. When TDPA is active, it modifies the desired velocities and enables the landing at around $45 \mathrm{~s}$. It is important to note that the red areas in the bottom plots correspond to a permanent interference of the passivity controller, as is usual in TDPA. The area is depicted as a filled color due to the high frequency switching action of the passivity controller, which is filtered out by the inner-loop controllers and does not affect the vehicles' motion.

Lastly, in order to analyze the action of the proposed 

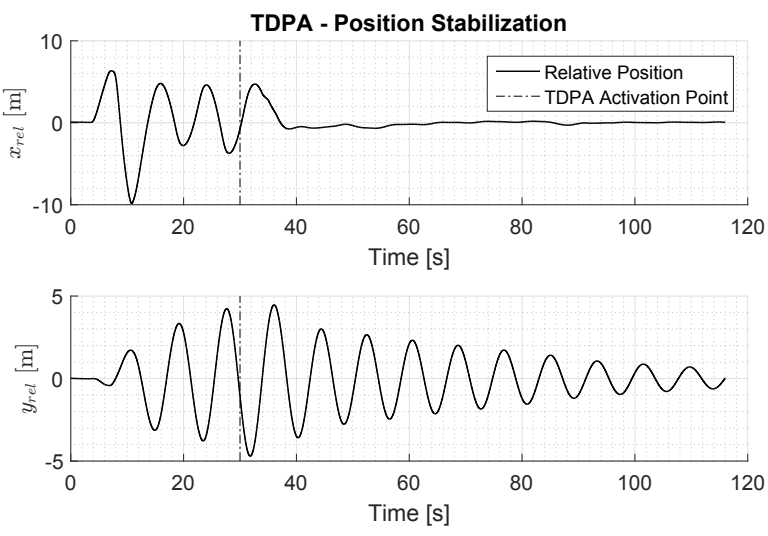

Fig. 11: Fail-safe behavior of the cooperative landing system for an excessive communication time delay. A round trip delay of $4 \mathrm{~s}$ and a crosswind gust are applied at around $5 \mathrm{~s}$ into the simulation to perturb the previously aligned vehicles. At around $30 \mathrm{~s}$ into the simulation (vertical dashed line) the passivity-controller (TDPA) is activated and stabilizes the system

approach in an extreme scenario, an analysis is performed in the horizontal plane, where the aircraft is flying at a constant altitude above the ground vehicle, while the vehicles are aligned in horizontal positions and velocities. In this case, since no vertical alignment is commanded, the safety mechanisms do not come into play and the vehicles are allowed to oscillate freely. Initially, no communication delay is present, then at around $5 \mathrm{~s}$, the communication time delay is increased to a very high value ( $4 \mathrm{~s}$ round trip delay) and a crosswind gust is applied to disturb the system. As can be seen in Fig. 11, the system starts to exhibit an unstable behavior after the crosswind gust event at around $5 \mathrm{~s}$ into the simulation. At around $30 \mathrm{~s}$ the passivity controller (TDPA) is manually activated. As can be seen in Fig. 11 stability is restored, where the relative longitudinal position quickly converges to small values while the lateral position takes longer to converge. The convergence time mainly depends on the damping ratio of the controlled vehicles. Overall, the passivity-based stabilizing controllers fulfill their design purpose and drive the system back to stability.

\section{CONCLUSiOnS}

This papers presented an energy-based control framework to allow for cooperative landings of fixed-wing UAVs on top of mobile platforms in the presence of communication delays. The effectiveness of the proposed method with respect to ensuring system stability was validated through numerical simulations for round-trip time delays of up to 4 seconds.

\section{REFERENCES}

[1] K. Lütjens, A. Lau, T. Pfeiffer, S. Loth, V. Gollnick, H. Klimek, B. Greßmann, S. Löwa, and C. Blank, Airport2030-Lösungen für den effizienten Lufttransport der Zukunft. Deutsche Gesellschaft für Luftund Raumfahrt-Lilienthal-Oberth eV, 2012.
[2] D. Rohacs, M. Voskuijl, and N. Siepenkotter, "Evaluation of landing characteristics achieved by simulations and flight tests on a smallscaled model related to magnetically levitated advanced take-off and landing operations," in ICAS 2014: Proceedings of the 29th Congress of the International Council of the Aeronautical Sciences, St. Petersburg, Russia, 7-12 September 2014. Citeseer, 2014.

[3] R. Tan and M. Kumar, "Tracking of ground mobile targets by quadrotor unmanned aerial vehicles," Unmanned Systems, vol. 2, no. 02, pp. 157173, 2014.

[4] S. Yang, J. Ying, Y. Lu, and Z. Li, "Precise quadrotor autonomous landing with srukf vision perception," in 2015 IEEE international conference on robotics and automation (ICRA). IEEE, 2015, pp. 21962201.

[5] T. Muskardin, G. Balmer, S. Wlach, K. Kondak, M. Laiacker, and A. Ollero, "Landing of a fixed-wing uav on a mobile ground vehicle," in 2016 IEEE International Conference on Robotics and Automation (ICRA). IEEE, 2016, pp. 1237-1242.

[6] A. Borowczyk, D.-T. Nguyen, A. Phu-Van Nguyen, D. Q. Nguyen, D. Saussié, and J. Le Ny, "Autonomous landing of a multirotor micro air vehicle on a high velocity ground vehicle," IFAC-PapersOnLine, vol. 50, no. 1, pp. 10488-10494, 2017.

[7] D. Falanga, A. Zanchettin, A. Simovic, J. Delmerico, and D. Scaramuzza, "Vision-based autonomous quadrotor landing on a moving platform," in 2017 IEEE International Symposium on Safety, Security and Rescue Robotics (SSRR). IEEE, 2017, pp. 200-207.

[8] T. Muskardin, G. Balmer, L. Persson, S. Wlach, M. Laiacker, A. Ollero, and K. Kondak, "A novel landing system to increase payload capacity and operational availability of high altitude long endurance uavs," Journal of Intelligent \& Robotic Systems, vol. 88, no. 2-4, pp. 597-618, 2017.

[9] A. Rodriguez-Ramos, C. Sampedro, H. Bavle, I. G. Moreno, and P. Campoy, "A deep reinforcement learning technique for visionbased autonomous multirotor landing on a moving platform," in 2018 IEEE/RSJ International Conference on Intelligent Robots and Systems (IROS). IEEE, 2018, pp. 1010-1017.

[10] D. Tzoumanikas, W. Li, M. Grimm, K. Zhang, M. Kovac, and S. Leutenegger, "Fully autonomous micro air vehicle flight and landing on a moving target using visual-inertial estimation and model-predictive control," Journal of Field Robotics, vol. 36, no. 1, pp. 49-77, 2019.

[11] J. Theis and F. Thielecke, "A generic rendezvous control solution for automatic landing of unmanned aircraft," in 5th CEAS Conference on Guidance, Navigation \& Control, 2019.

[12] D. Lee, T. Ryan, and H. J. Kim, "Autonomous landing of a vtol uav on a moving platform using image-based visual servoing," in 2012 IEEE international conference on robotics and automation. IEEE, 2012, pp. 971-976.

[13] T. Muskardin, "Autonomous landing of fixed-wing aircraft on mobile platforms," Ph.D. dissertation, Escuela Técnica Superior de Ingeniería, University of Seville, Spain, 2020.

[14] J. Artigas, R. Balachandran, C. Riecke, M. Stelzer, B. Weber, J.-H. Ryu, and A. Albu-Schaeffer, "Kontur-2: force-feedback teleoperation from the international space station," in 2016 IEEE International Conference on Robotics and Automation (ICRA). IEEE, 2016, pp. 1166-1173.

[15] J.-H. Ryu, J. Artigas, and C. Preusche, "A passive bilateral control scheme for a teleoperator with time-varying communication delay," Mechatronics, vol. 20, no. 7, pp. 812-823, 2010.

[16] A. Lambregts, "Vertical flight path and speed control autopilot design using total energy principles," in Guidance and Control Conference, 1983 , p. 2239.

[17] B. Hannaford and J.-H. Ryu, "Time-domain passivity control of haptic interfaces," IEEE Transactions on Robotics and Automation, vol. 18, no. 1, pp. 1-10, 2002.

[18] J. Artigas, J.-H. Ryu, and C. Preusche, "Position drift compensation in time domain passivity based teleoperation," in 2010 IEEE/RSJ International Conference on Intelligent Robots and Systems. IEEE, 2010, pp. $4250-4256$.

[19] A. Coelho, H. Singh, T. Muskardin, R. Balachandran, and K. Kondak, "Smoother position-drift compensation for time domain passivity approach based teleoperation," in 2018 IEEE/RSJ International Conference on Intelligent Robots and Systems (IROS). IEEE, 2018, pp. 5525-5532.

[20] A. Coelho, C. Ott, H. Singh, F. Lizarralde, and K. Kondak, "Multi-dof time domain passivity approach based drift compensation for telemanipulation," in 2019 19th International Conference on Advanced Robotics (ICAR), 2019, pp. 695-701. 